\title{
Volume Manufacturing of Hollow Core Photonic Band Gap Fibers: Challenges and Opportunities
}

\author{
G. T. Jasion', F. Poletti ${ }^{1}$, J. S. Shrimpton², D. J. Richardson ${ }^{1}$ \\ ${ }^{1}$ Optoelectronics Research Centre, University of Southampton, SO17 1BJ, UK \\ ${ }^{2}$ Faculty of Engineering and the Environment, University of Southampton, SO17 1BJ, UK \\ g.jasion@soton.ac.uk
}

\begin{abstract}
A fluid dynamics model is used to investigate yield increase in hollow-core bandgap fibers. $100 \mathrm{~km}$ of good fiber from one preform seems feasible; for higher yields some observed distortions would need to be addressed.

OCIS codes: (000.4430) Numerical approximation and analysis; (060.2280) Fiber design and fabrication; (060.5295) Photonic crystal fibers;
\end{abstract}

\section{Introduction}

Due to their ultralow nonlinearity and latency, Hollow Core Photonic Band Gap Fibers (HC-PBGFs) are a potential candidate for next generation telecoms fibers [1]. Current research production of HC-PBGF yields relatively short, $\mathrm{km}$ scale, and consequently pricey fibers. If this technology is ever to replace conventional fibers in at least selected telecoms applications, the capacity to volume-produce HC-PBGFs in lengths exceeding $100 \mathrm{~km}$ needs to be developed. In this paper we use a recently developed fluid dynamics model [2,3] to virtually study, for the very first time, the draw of fibers up to $500 \mathrm{~km}$ long. The challenges to be faced in order to achieve a good microstructure, and therefore guidance properties, are discussed.

HC-PBGFs are fabricated using a 2 stage stack and draw process with a typical second stage preform being approximately 1 meter long, and less than $2 \mathrm{~cm}$ in diameter, which can yield approximately $5 \mathrm{~km}$ of fiber. To increase the yield the preform needs to contain more glass, and for practical manual handling it is preferable not to increase the length of preform, but instead to enlarge its diameter and to modify the drawing parameters accordingly.

In this work we start by simulating a known HC-PBGF with the potential to yield $5 \mathrm{~km}$ of fiber that has been recently produced experimentally. We then work towards increasing the yield by enlarging the cross-sectional dimensions of the preform while altering the draw conditions to achieve the same final fiber geometry. We report the simulated structure obtained for 5 progressively larger preforms and observe that a 20 times increase in yield from current state-of-the-art seems entirely realistic. For even longer fiber yields some distortions are observed and discussed, we conclude with some recommendations to avoid them.

\section{Methodology}

To predict the fiber draws in this study we used the MicroStructure Element Method (MSEM) [2]; a recent numerical model for the simulation of microstructured fiber draws that has been demonstrated to accurately reproduce the draw of an HC-PBGF fiber with hundreds of holes [3]. The MSEM discretizes the geometry and then it applies fluid forces (viscosity, pressure and surface tension) to evolve the structure through the draw.

In this study we investigate 5 preforms, all designed to produce the same 19 cell core, 7 ring of holes HC-PBGF guiding at $1550 \mathrm{~nm}$, that was fabricated and simulated in [3]. The fiber has an outer diameter of $160 \mu \mathrm{m}$, a microstructured cladding diameter of $80 \mu \mathrm{m}$ and a hole-to-hole spacing of $\sim 4 \mu \mathrm{m}$. All preforms are $1 \mathrm{~m}$ long; the initial preform (a) has a total yield of $\sim 5 \mathrm{~km}$, which increases to 10, 50, 100 and $500 \mathrm{~km}$ for fibers (b)-(e), respectively. The dimensions of the preforms are given in Table 1. The feed speed of the preform into the furnace is kept constant at $2 \mathrm{~mm} / \mathrm{min}$, giving in all cases a total drawing time of approximately 8 hours.

Table 1. Preform dimensions and draw conditions. The shaded cells indicate the largest pressure difference achievable when targeting the ideal core size, see Fig. 2.

\begin{tabular}{|c|c|c|c|c|c|c|c|}
\hline & $\begin{array}{l}\text { Yield } \\
(\mathrm{km})\end{array}$ & $\begin{array}{c}\text { Jacket OD } \\
(\mathrm{mm})\end{array}$ & $\begin{array}{c}\text { Jacket ID } \\
(\mathrm{mm})\end{array}$ & $\begin{array}{c}\text { Cane strut } \\
\text { thickness }(\mu \mathrm{m})\end{array}$ & $\begin{array}{c}\text { Furnace Peak } \\
\text { Temp. }{ }^{\circ} \mathrm{C} \\
\end{array}$ & $\begin{array}{c}\Delta \mathbf{P} \text { Core - cladding } \\
(\mathrm{kPa})\end{array}$ & $\begin{array}{c}\text { Draw speed / Feed } \\
\text { speed }\end{array}$ \\
\hline $\mathbf{a}$ & 5 & 10.6 & 4.0 & 44 & 1752 & -8.3 & $5 \times 10^{3}$ \\
\hline b & 10 & 14.8 & 5.2 & 62 & 1806 & -6.7 & $10 \times 10^{3}$ \\
\hline c & 50 & 32.7 & 10.5 & 140 & 1925 & -4.4 & $50 \times 10^{3}$ \\
\hline d & 100 & 46.1 & 14.4 & 200 & 1981 & -3 & $100 \times 10^{3}$ \\
\hline e & 500 & 102.8 & 31.0 & 440 & 2120 & -1.3 & $500 \times 10^{3}$ \\
\hline
\end{tabular}




\section{Drawing Parameters}

Increasing the size of the preforms results in much faster fiber draw speeds. In order to maintain a practical value of tension, which reduces the chance of fiber breaks (here: $200 \mathrm{~g}$ was used in all cases), we chose to increase the furnace temperature. The longitudinal temperature profile of the furnace is modelled as Gaussian; the furnace length $(8 \mathrm{~cm})$, entrance and exit temperatures are fixed across all cases, Fig. 1.

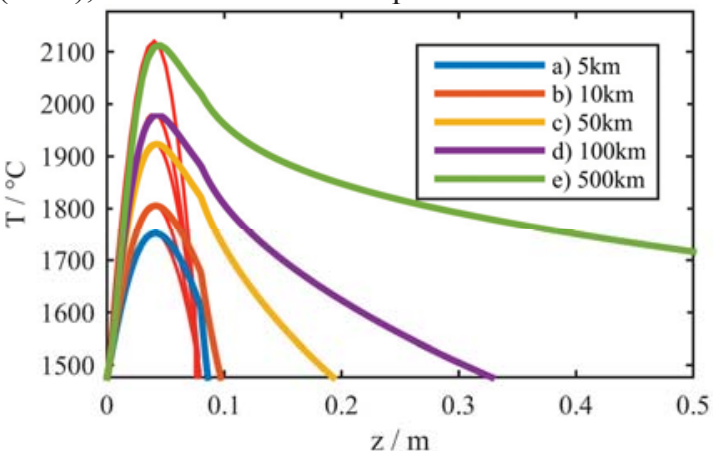

Fig. 1: Furnace (red) and fiber (color) temperature profiles, used for the different preforms. The fiber continues to change until it is cool.

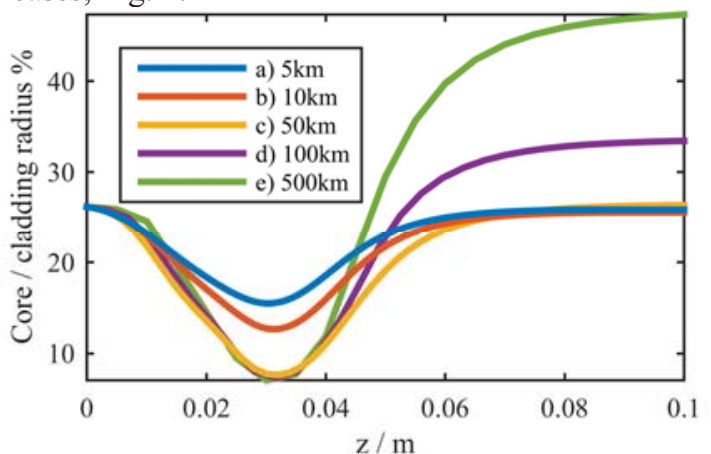

Fig. 2: Core size during the draw, core pressure causes an over contraction of the core $(<0.3 \mathrm{~m})$, core then expands under surface tension. Applicable pressure is limited by core collapse ( $7 \%$ radius) at $0.03 \mathrm{~m}$.

The increase in physical dimension and temperature requires that the difference between core and cladding pressure must be adapted. The fiber we are targeting has an ideal core to cladding ratio of $26 \%$ (based on preserving the initial shape of the structure); the core pressure in these draws has been adjusted to achieve this where possible. We observe that the applied negative core pressure causes the core to contract initially, until the geometry has reduced in size at which point the surface tension starts to dominate, causing the core to slowly expand back, Fig. 2. Note that in fibers $(\mathrm{d}, \mathrm{e})$ the core ratio could not be maintained at $26 \%$. In these cases the core pressure shown in Table 1 is the largest difference that we could apply that would not result in a completely collapsed core at mid-draw (at $\sim 3.5 \mathrm{~cm}$ from the furnace entrance).

\section{Results}

Figure 3 shows the geometry of the final 5 fibers that we were able to obtain, with increasing yield from 5 to 500 $\mathrm{km}$.

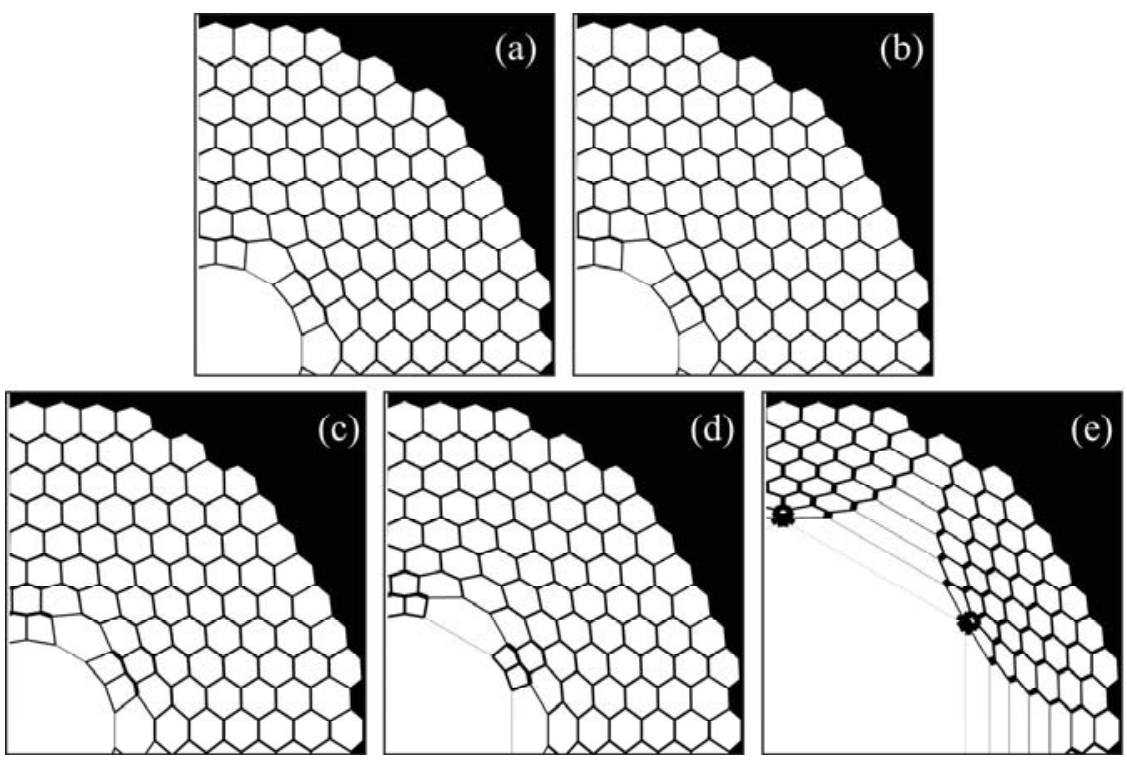

Fig.3. Final structure of fiber for each of the preforms in Table 1.

The structures for fibers $(\mathrm{a}-\mathrm{c})$ are very close to the optimum desired target. Fiber (d), i.e. $100 \mathrm{~km}$ draw, shows some sign of distortion, similar to the fiber in [1], which is not ideal but which could probably be tolerable, while in the $500 \mathrm{~km}$ case (e) the distortions become excessive for good optical performance. To understand why this has 
happened we need to look at the axial temperature profile of the fibers, Fig. 1. The higher yield preforms have much faster fiber speeds, this extends the cooling period in time and $z$; the outcome of this is that there is a significant time during which the surface tensions acts on the structure causing the core to expand. For the smaller preforms this is easily counterbalanced by the application of a negative pressure difference between the core and the cladding, however, the pressure is only effective where the structure is large; when the geometry becomes smaller, later on in the neck down, the surface tension dominates. For the larger preforms the higher temperature used reduces the viscosity and the surface tension is then able to expand the core more, with less resistance, as shown in Fig. 2.

The core overexpansion is further complicated by an additional distortion due to the interplay between surface tension and pressure in the early phase of the draw, Fig. 4. The surface tension is not perfectly normal to the core boundary; its azimuthal component is normally limited by the viscous force along the struts at the core wall, but at the higher temperatures used for the large preforms in this study, the viscosity is too low and the structure deforms easily. These two deformation mechanisms, expanding core and deforming structure, are both a symptom of low viscosity in the microstructure.

If the peak temperature of the preform can be reduced then the structure will improve because the viscous forces would be large enough to prevent the distortion seen in Fig 4. In practice however, reduction in temperature leads to increased likelihood of fiber breaks, this limitation will need to be overcome first.

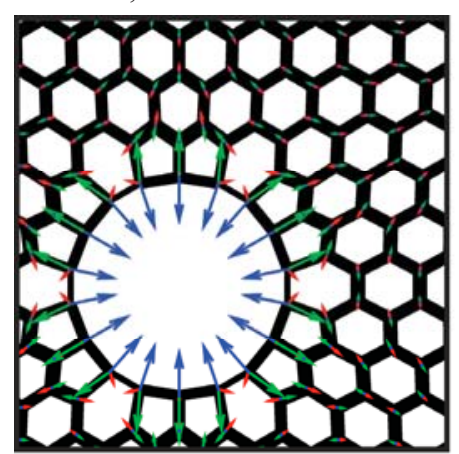

(a)

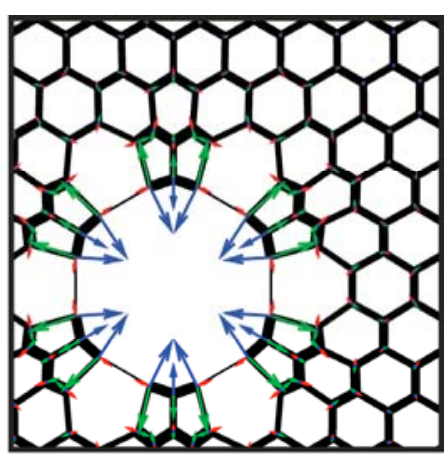

(b)

Fig 4. Fiber (e), $500 \mathrm{~km}$ yield, at $0.02 \mathrm{~m}$ (a) and $0.025 \mathrm{~m}$ (b). Pressure forces shown by blue arrows, surface tension green and viscosity red. The increased temperature reduces the viscosity so it is unable to prevent the rapid distortions caused by the surface tension.

\section{Thermal assumptions}

The peak furnace temperature in our model has been tuned to ensure that the fiber tension (function of the glass viscosity) is equal to $200 \mathrm{~g}$, this often results in a low furnace temperature. It is important to recreate the glass dynamics, therefore we consider the tension a more appropriate metric to match from experiment to simulation. The thermal model used in the simulation contains some simplifications and assumes that temperature is radially uniform. The impact of this assumption increases with the diameter of the preform; in general, the absorption of thermal radiation by the glass will result in a radial temperature gradient, whereby thermal conduction may become a significant component of heat transfer to the glass in the center of the preform. In practical terms this may have a favorable outcome, as low temperatures in the center of the microstructure will lead to more uniform structures as demonstrated in Fig. 3, but more investigation is required.

\section{Conclusions}

For the first time we have investigated the possibility to increase the fiber yield of HC-PBGFs. We have found that acceptable structures could be achieved in fiber draws of up to $100 \mathrm{~km}$ without any particular modification of the drawing process, which is promising from a yield and cost reduction point of view. The $500 \mathrm{~km}$ sample was found to undergo dramatic distortions, the cause of which was explained. Mitigating strategies to enable even longer fiber draws by increasing the viscosity in the microstructure will be the subject of future studies.

This work was supported by the EU 7th Framework Programme under grant agreement 258033 (MODE-GAP) and by the Royal Society.

\section{References}

[1] Poletti, F., et al. "Towards high-capacity fibre-optic communications at the speed of light in vacuum." Nature Photonics 7.4, 279-284 (2013).

[2] Jasion G. et al., "Novel Fluid Dynamics Model to Predict Draw of Hollow Core Photonic Band-Gap Fibres," in Optical Communications (ECOC), 2042 40th European Conference and Exhibition on, Th. 2.4.3, Cannes, France, 21 - 25 Sep. 2014.

[3] Jasion G. T. et al., "MicroStructure Element Method (MSEM): Viscous Flow Model for the Virtual Draw of Microstructured Optical Fibers," Optics Express, manuscript in preparation. 